\title{
A new neutron monitor at the Juan Carlos I Spanish Ant- arctic Station (Livingston Island-Antarctic Peninsula)
}

\author{
Juan José Blanco ${ }^{\circledR 1}$, Óscar García Población ${ }^{\circledR 1}$, Juan Ignacio García Tejedor ${ }^{\circledR 1}$, Sindulfo Ayuso ${ }^{\circledR 1}$, \\ Alejandro López-Comazzi ${ }^{\circledR 1}$, Iván Vrublevskyy ${ }^{\circledR 1}$, Christian T. Steigies ${ }^{\circledR 2}$ \\ Correspondence \\ 'CaLMa - Monitor de Neutrones de Castilla-La Mancha, Universidad de Alcalá, Madrid, Spain, juanjo.blanco@uah.es \\ ${ }^{2}$ Extraterrestrial Physics, Institute of Experimental and Applied Physics, Kiel University, Germany
}

\section{OPEN ACCESS}

This work is published under the Creative Commons Attribution 4.0 International licence (CC BY 4.0). Please note that individual, appropriately marked parts of the work may be excluded from the licence may be excluded from the licence other copyright conditions. other copyright conditions. If such thirdparty material is not under the Creative Commons license any copying, editing or public reproduction is only permitted with the prior consent of the respective copy right owner or on the basis of relevant legal authorization regulations.

\section{Keywords}

cosmic ray; neutron monitor instrumentation

\begin{abstract}
Last January 2019, a new neutron monitor was installed at Juan Carlos I Spanish Antarctic Station (62 $39^{\prime} 46^{\prime \prime}$ S, $60^{\circ} 23^{\prime} 20^{\prime \prime} \mathrm{W}, 12 \mathrm{~m}$ asl) located in Livingston Island (South Shetland Archipelago) close to the Antarctic Peninsula. The vertical rigidity cut-off for this new station is estimated as $3.52 \mathrm{GV}$. This new station (ORC) is composed of a BF3-based 3NM64 (ORCA) and 3 bare BF3 counters (ORCB). The neutron monitor is complemented by a muon telescope sharing a common room in a single stack. ORCA and ORCB with the Castilla-La Mancha neutron monitor ( $\mathrm{CaLMa}$ ) are the Spanish contributions to the Neutron Monitor Data Base. Because Juan Carlos I station is a summer station, one minute data is providing once a day during the Antarctic summer. One hour data are sent once a day during Antarctic winter. First measurements and future plans are provided in this work.
\end{abstract}

\section{Introduction}

Neutron monitors are not uniformly distributed around the World. The stations are concentrated mostly on the Northern hemisphere and Antarctica. Each neutron monitor is characterized by its altitude above sea level (asl) and vertical cut-off rigidity. Two of these neutron monitors are maintained by the Space Research Group of Alcalá University. One of then, CaLMa, is operating since mid 2012 in Guadalajara (Spain) 40³8' N, 3 3 $9^{\prime} \mathrm{W}$ and $708 \mathrm{~m}$ above sea level (Medina et al. 2013). The second one, ORCA, has been recently installed (January 2019) at Juan Carlos I Spanish Antarctic Base (BAE). Both neutron monitors are part of the Neutron Monitor Data Base (NMDB 2009) although ORCA is not providing data yet.

\section{Antarctic Cosmic Ray Observatory (ORCA)}

\subsection{ORCA location}

Juan Carlos I Spanish Antarctic Base (BAE) is a summer research base located in Livingston Island, South Shetland Islands, Maritime Antarctica 62 ${ }^{\circ} 39^{\prime} 46^{\prime \prime} \mathrm{S}, 60^{\circ} 23^{\prime} 20^{\prime \prime} \mathrm{W}$, and $12 \mathrm{~m}$ asl. The location of the base and the penumbra analysis is shown in figure 1 . The rigidity values at Juan Carlos I Spanish Antarctic Base on 2019-02-02 12:00:00 UT are $\mathrm{R}_{\mathrm{L}}=2.221 \mathrm{GV}, \mathrm{R}_{\mathrm{U}}=2.673 \mathrm{GV}$ according to the results of the calculator at https://tools.izmiran.ru/ (last accessed May 31, 2021) using the IGRF 
model. For a flat spectrum, the effective rigidity of geomagnetic cutoff is $\mathrm{R}_{\text {eff }}=2.487 \mathrm{GV}$. Its location, close to the Antarctic Peninsula, covers a gap in the global distribution of neutron monitors. LARC neutron monitor (Cordaro et al. 2012) was operative at King George Island, 134 km apart from Livingston Island, covering such gap in the neutron monitor global network but it is switched off nowadays. Close to the scientific module in BAE Juan Carlos I, in a thermally insulated container, the Antarctic Cosmic Ray Observatory (ORCA) was installed at the beginning of January 2019.

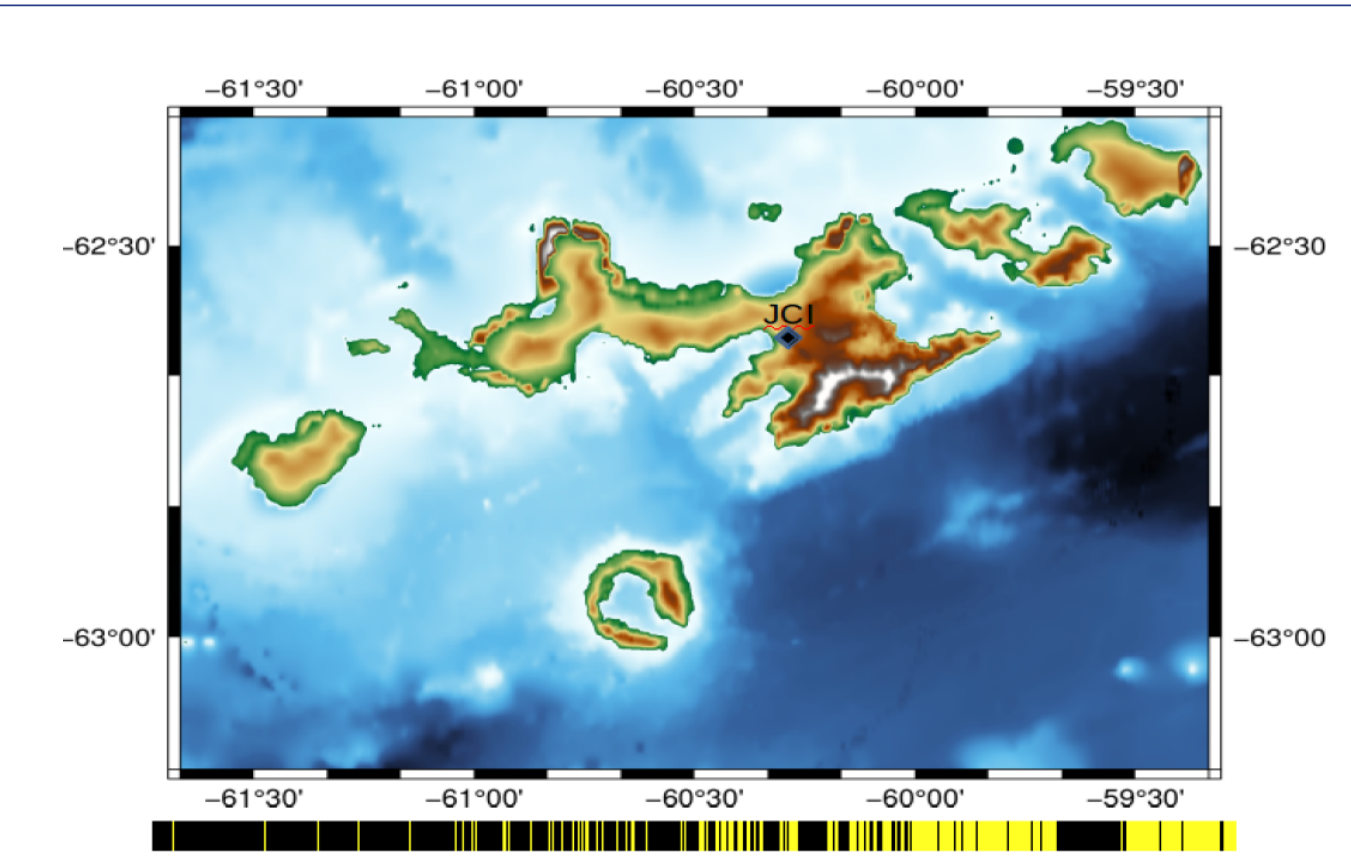

Figure 1: Map of Juan Carlos I location at Livingston Island with Penumbra at date 2019-01-02 12:00:00 UT. $\mathrm{RL}=2.221 \mathrm{GV}$ and $\mathrm{RU}=2.673 \mathrm{GV}$.

\subsection{ORCA description}

The Antarctic Cosmic Ray Observatory (ORCA) is an instrument to monitor the cosmic ray flux by the observation of secondary cosmic rays at ground level. It was designed to be operated at Juan Carlos I Spanish Antarctic Base and is made by a set of detectors with different targets and capabilities. ORCA is composed of a neutron detector (NEMO) and muon telescope (MITO) installed in a 20 feet container which is thermally isolated and environmentally controlled (figure 2).

The Neutron Monitor (NEMO) is composed of two groups of three proportional detectors. The counters in the first set are BP-28 detectors (NEMO-3NM64) and follow the NM64 standard, i. e. an outer reflector made of polyethylene, with lead producers in the form of lead rings around the moderator, a moderator also of polyethylene and the counter tube surrounded by the moderator. The second set is integrated by three bare, i. e. without a lead producer or polyethylene, LND2061 counters (NEMO-3BNM). NEMO-3NM64 and NEMO-3BNM measure neutrons at two different energy thresholds (bare versus lead surrounding counters). The signals from the proportional counter are preprocessed in a signal conditioning system before reaching the data acquisition system. This system consists of an FPGA with an IP core specifically designed for this application, and an embedded Linux Beaglebone Black system in which the capture code is executed, as well as the necessary corrections, the editing of the data and its subsequent publication in a database (Población et al. 2014). This system keeps its local clock synchronized using an NTP server equipped with a 
GPS receiver. Additionally, it also controls a Vaisala meteorologic station PTU301 Transmitter which provides pressure, temperature and relative humidity. The temperature/humidity probe composed of a Pt100 RTD Class F0.1 IEC 60751 and Vaisala HUMICAP 180C respectively (table 1).

The Muon Impact-Tracer Observer (MITO) is a telescope made by a stack of two (MITO-top and MITO-bottom) BC-400 organic scintillators $(100 \mathrm{~cm}$ x $100 \mathrm{~cm}$ x $5 \mathrm{~cm}$, poly-vinyl-toluene with $65 \%$ anthracene). Four photomultipliers (PMTs) are coupled to each scintillator by means of a pyramidal light guide. Each PMT collects the light reaching the lateral surface and generates a pulse which carries information about the distance between the particle impact point and the corresponding lateral surface of the BC-400 scintillator. Track impact point is calculated by comparison of the pulse height detected in the PMTs when they are working in coincidence. The two scintillators, impact points give us information about the muon incident direction. Both scintillators are placed at the top and bottom of a metallic structure 136,5 $\mathrm{cm}$ apart from each other with a $10 \mathrm{~cm}$ lead layer in between (figure 3 ).

MITO has two data acquisition systems operating in parallel, SAS and Aracne. SAS counts the impacts on the scintillator keeping those that follow four different coincidence channels, Top: the four PMTs in the upper scintillator, Bottom: the four PMTs in the bottom scintillator, coin8: the eight PMTs, i.e. particles that cross both scintillators, and Lateral: a combination of two PMTs in the upper scintillator located at a common lateral side and two PMTs in the bottom scintillator but at the opposite lateral side of the previous ones. Aracne records the highest pulse of all the PMTs gathered in particle detection. From them, it can select the events under the same coincidence channels as SAS. The pulse height analysis allows one to determine the impact point on the scintillators and with the two impact points, the particle trajectory throughout MITO can be reconstructed. MITO is operating in a one minute counting mode with four coincidence channels and an event to event storage of the pulse height recorded in every PMT simultaneously with a time resolution of $25 \mathrm{~ns}$.

\begin{tabular}{|c|c|c|}
\hline & NEMO 3NM64(ORCA) & NEMO 3BNM(ORCB) \\
\hline Counter Type & BP28 & LND2061 \\
\hline Effective diameter (mm) & 148.5 & 149.1 \\
\hline Effective length (mm) & 1908.0 & 1956.3 \\
\hline Cathode material & Stainless steel & Stainless steel \\
\hline Gas filling & $\mathrm{BF}_{3}\left(96 \%{ }^{10} \mathrm{~B}\right)$ & $\mathrm{BF}_{3}\left(96 \%{ }^{10} \mathrm{~B}\right)$ \\
\hline Gas pressure (mmHg) & 200 & 200 \\
\hline \multirow[t]{2}{*}{ Operational voltage (V) } & -2700 & 1800 \\
\hline & MITO Top & MITO Bottom \\
\hline Scintillator & $\mathrm{BC} 400$ & BC400 \\
\hline Dimension $(\mathrm{cm})$ & $100 \times 100 \times 5$ & $100 \times 100 \times 5$ \\
\hline Operational voltage (V) & $1200-1400$ & 1000 \\
\hline \multirow[t]{2}{*}{ PMT } & $4 \mathrm{R} 2154$ & $4 \mathrm{R} 2154$ \\
\hline & Vaisala Meteorologic station & \\
\hline PTU 301 & $500-1100 \mathrm{hPa}$ & $\pm 0.05 \mathrm{hPa}$ \\
\hline Pt100 & -40 to $60^{\circ} \mathrm{C}$ & $\pm 0.2^{\circ} \mathrm{C}$ \\
\hline HUMICAP 180C & $0-100 \%$ & $\pm 1 \%$ \\
\hline
\end{tabular}

Table 1: ORCA instruments. 


\subsection{Data handling}

ORCA is measuring with a cadence of one minute as a general approach. Neutrons, muons and environmental values, pressure, temperature and humidity, are recorded with a temporal resolution of one minute. Additionally, MITO can work in a specific mode to acquire the complete pulse shape during a time interval. The duration of this interval depends on the amount of data that can be stored in every moment because for every particle incoming on MITO volume eight complete pulse signals are gathered.

Throughout the period when Juan Carlos I Base is open (Antarctic summer), real time data are sent to a web server and also are stored in a massive hard disc. Nevertheless, no real time data can be sent in winter when the base is closed although a set of data can be gathered once a month remotely.

\subsection{Atmospheric corrections}

NEMO and MITO measurements, i.e. secondary neutrons and muons, are strongly affected by the air pressure, the more pressure the less counting rate, and the usable counting rate value has to be corrected taking into account this fact. The correction factor $\beta$ is obtained fitting the counting rate to an exponential law of pressure. From the fits for the 3NM64 group and the 3BNM group the $\beta_{A}=0.00735 \mathrm{hPa}^{-1}, \beta_{B}=0.00639 \mathrm{hPa}^{-1}$ for $3 \mathrm{NM} 64$ and $3 \mathrm{BNM}$ respectively. The result of the fit procedure is shown in figures 5 and 6.

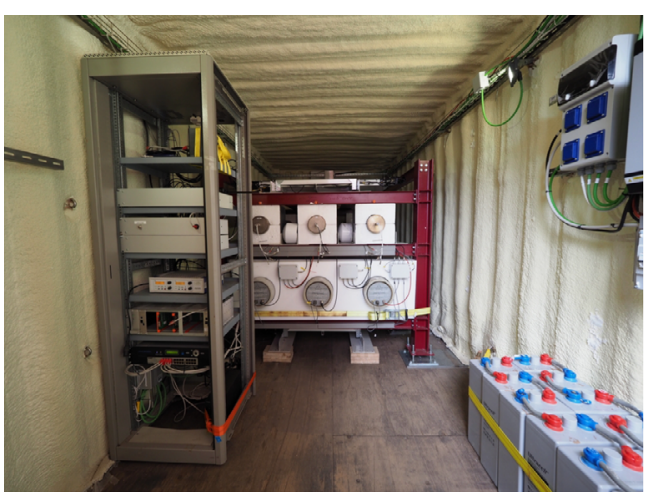

Figure 3: ORCA configuration into the maritime container.

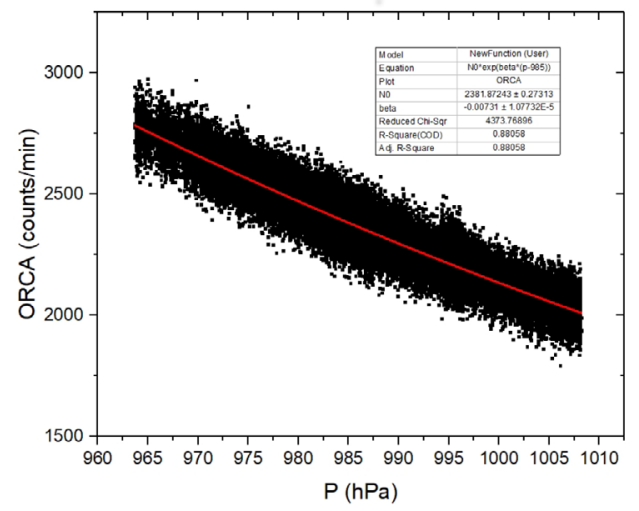

Figure 5: 3NM64 pressure correction.

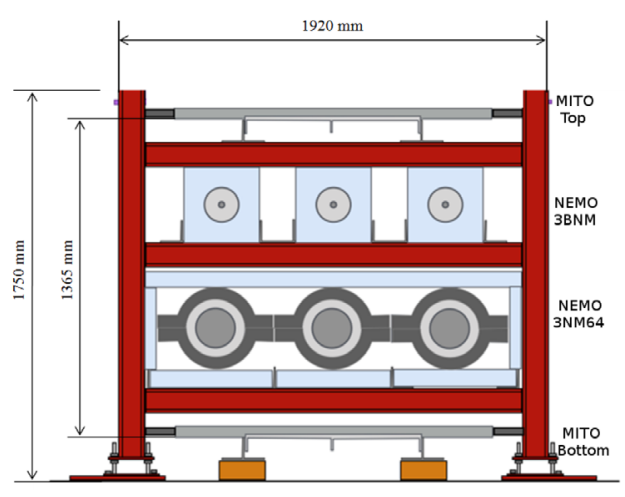

Figure 4: NEMO and MITO layout.

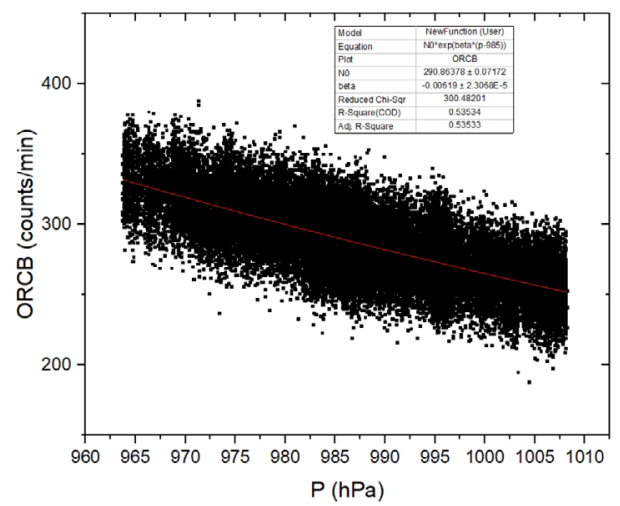

Figure 6: 3BNM pressure correction. 
A similar procedure is performed for the MITO coincidence modes, i. e., Top, Bottom, Coin8 and Lateral being the obtained factor, $0.00234 \mathrm{hPa}^{-1}, 0.00177 \mathrm{hPa}^{-1}, 0.00179 \mathrm{hPa}^{-1}$ and $0.00141 \mathrm{hPa}^{-1}$, respectively.

\subsection{Data product}

ORCA can observe neutrons and muons in a continuous way giving counting rates with a maximum temporal resolution of one minute. Real time data can be produced while the Juan Carlos I base is operative and once a month during the Antarctic winter. Muon impact point and incoming

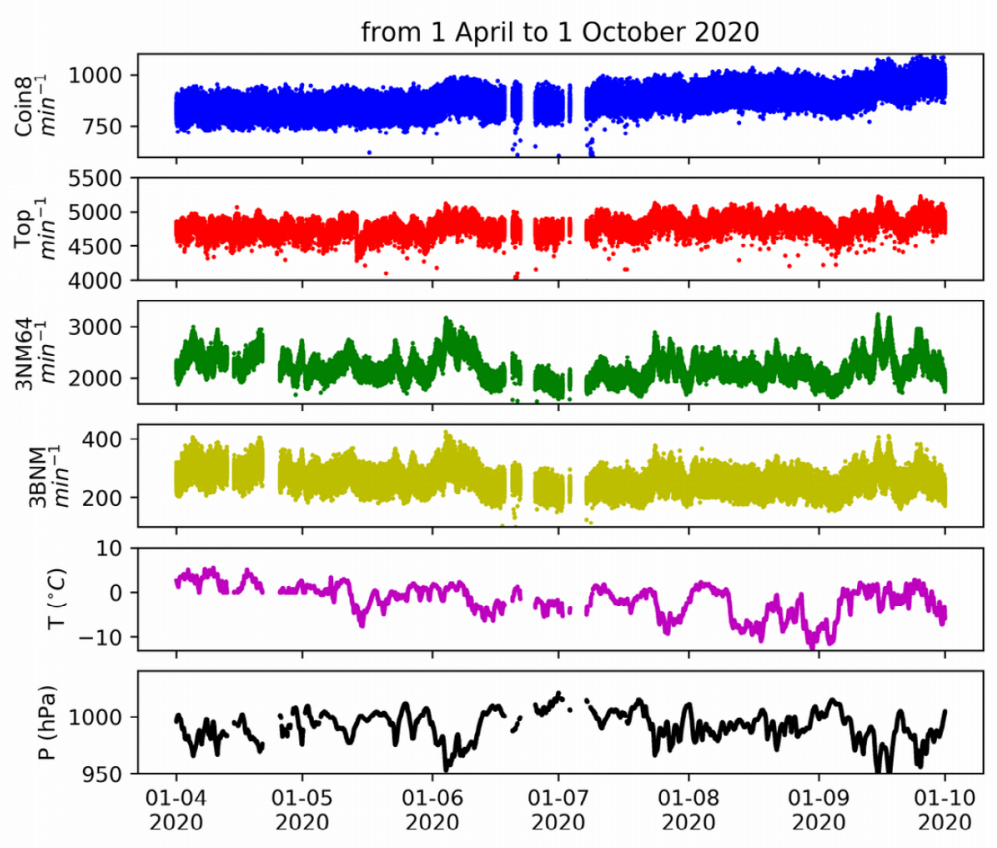

Figure 7: NEMO and MITO counting rates, temperature, pressure and humidity along the Antartic winter.

direction are also produced. Examples of this are shown in figures 7 and 8. Data was collected throughout the winter except for two weeks when renewable energy was discontinued. Pressure uncorrected counting rates are presented from two MITO coincidence modes and NEMO-3NM64 and NEMO-3BNM. Temperature inside the container and pressure are also shown.

\section{Conclusions}

ORCA is in nominal phase since January 2019 at Juan Carlos I Antartic Base in Livingston Island (Antarctica) at a rigidity cut-off of $2.487 \mathrm{GV}$. It can provide real time data when Juan Carlos I Base is open (Antarctic summer) and download data once a month when the Base is closed.

Neutron counting rates at two different energy thresholds are provided by 3NM64 and 3BNM modules. Muon counting rates and incident directions are measured by MITO, a new muon telescope design with minimum power consumption and maintenance.

ORCA is expected to be part of the NMDB with the following identifiers ORCA for the 3NM64 module and ORCB for the bare counters module (3BNM). 


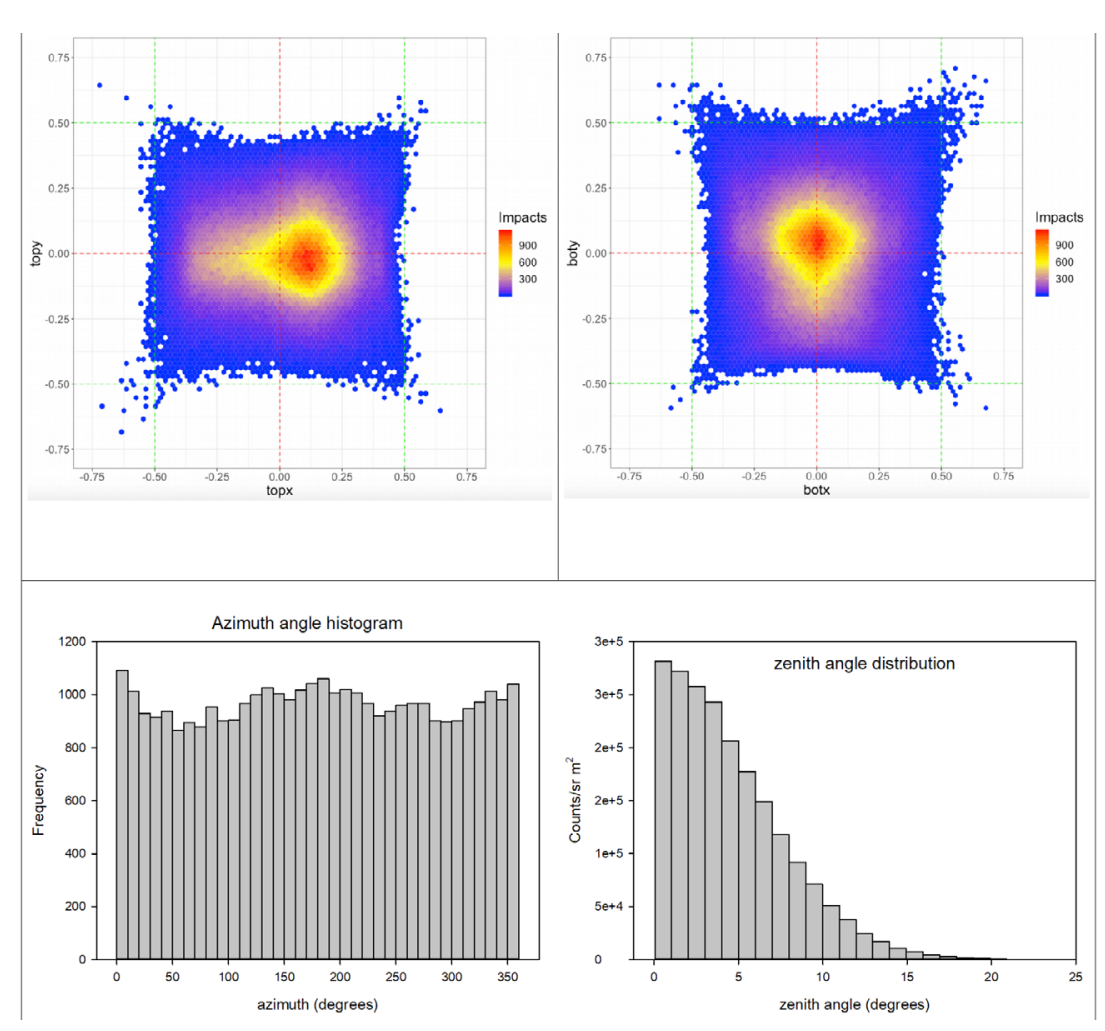

Figure 8: Top left and top right impact point on MITO Top and MITO Bottom respectively. Bottom, azimuth and zenith of incoming tracks thorught MITO.

\section{Acknowledgments}

Thanks to project CTM2016-77325-C2-1-P funded by Ministerio de Economía y Competitividad and by the European Regional Development Fund, FEDER.

\section{References}

Cordaro, E.G., Olivares, E., Galvez, D., Salazar-Aravena, D., and Laroze, D., 2012, Advances in Space Research, 49(12):1670-1683 Medina, J., Blanco, J. J., García, O., Gómez-Herrero, R., Catalán, E. J., García, I., Hidalgo, M. A., Meziat, D., Prieto, M., Rodríguez-Pacheco, J., and Sánchez, S., 2013, Nucl. Instrum. Methods Phys. Res. A, 727:97-103, DOl: https://doi.org/10.1016/j.nima.2013.06.028

Población, Ó. G., Blanco, J. J., Gómez-Herrero, R., Steigies, C. T., Medina, J., Tejedor, I. G., and Sánchez, S., 2014, Journal of Instrumentation, 9(08):T08002-T08002, DOI: https://doi.org/10.1088/1748-0221/9/08/t08002

Neutron Monitor Database, 2009, https://www.nmdb.eu/(last accessed September 11, 2020) 